\title{
Performance of First and Second Order Sliding Mode Control Strategies for a Simple Pendulum
}

\author{
${ }^{* 1}$ B.Amarendra Reddy, ${ }^{2}$ B.Pavan Kumar, ${ }^{3}$ G.Ananda Rao, ${ }^{4}$ K. Anusha \\ 1, 2,3,4 Department of Electrical Engineering, Andhra University(A) \\ Email: bamarendrareddy@yahoo.com
}

\section{Received: 10 $^{\text {th }}$ December 2017, Accepted: 17th January 2018, Published: 28th February 2018}

\begin{abstract}
Sliding mode control approach is recognized as one of the efficient tools to design robust controllers for complex high-order dynamic plant operating under uncertainty conditions. The main advantages of sliding mode are low sensitivity to plant parameter variations and disturbances can be completely eliminated. This paper deals with the modeling and control of simple pendulum with different SMC strategies under various perturbed conditions. Here, first-order sliding mode control and second-order sliding mode control strategies are considered for regulation of simple pendulum. Control of simple pendulum, under different initial conditions is demonstrated in simulation in MATLAB environment.
\end{abstract}

Keywords: Simple Pendulum, Sliding Mode Control, First Order Sliding Mode Control, Second Order Sliding Mode Control (Twisting and Super Twisting )

\section{Introduction}

A simple pendulum system is a mechanical system that exhibits periodic motion. It consists of a particle like bob suspended by a light string that is fixed at the upper rigid surface. The motion occurred in the vertical plane is driven by its weight [1].Pendulums have been known to be a good resonators and also suitable for reference in high quality clocks for a long time. In this paper a robust control strategy to control the oscillations of a simple pendulum was designed. This can be achieved by using Sliding Mode Control (SMC) which is one of the Variable Structure Control Systems (VSCS).

Variable structure control systems, as the name suggests, these are a class of systems where the 'control law' is deliberately changed during the control process according to some predefined rules, which depend on the states of the system. Variable Structure Control Systems have been described in the literature by numerous authors [2]-[5]. The most distinguished feature of variable structure control (VSC) is its ability towards robust control systems and using VSC in many cases results in invariant control systems. The term "invariant" means that the system is completely insensitive to parametric uncertainty and external disturbances.

The basic idea of sliding mode control is that the structure of the control signal is altered as the state trajectory crosses switching surfaces in the state space as a result the desirable properties are achieved. SMC design entails the choice of the switching control and the associated switching logic to yield sliding motion on the sliding surface. Although sliding mode control method provides a robust controller for nonlinear dynamic systems [4][6] and it inherits a discontinuous control action forces the system to reach the sliding surface.

This paper is organized as follows. (i) Mathematical modeling aspects of the Simple Pendulum is discussed in the section II. (ii) Sliding mode Control law aspects and Control strategy are discussed in section III. (iii) Simulation studies using MATLAB are discussed in section IV. (iv) Conclusions are presented in section $\mathrm{V}$.

\section{MATHEMATICAL MODELLING}

Consider the simple pendulum shown in Fig. 1, where 1 denotes the length of the rod and $\mathrm{m}$ denotes the mass of the bob. Assume the rod is rigid and has zero mass.

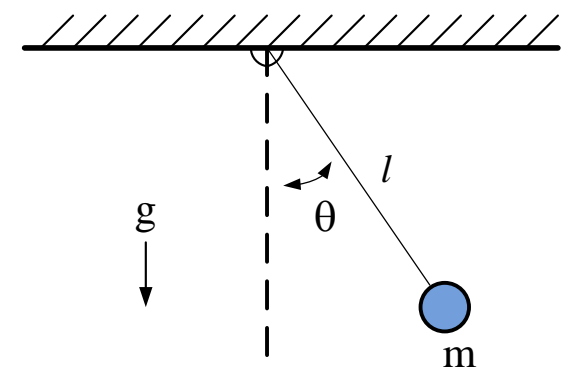

Fig. 1. Simple Pendulum

Where $\theta$ denote the angle subtended by the rod and the vertical axis through the pivot point. The pendulum is free to swing in the vertical plane. To write the mathematical equation of motion of the pendulum, we need to identify the forces acting on the bob. There is a downward gravitational force equal to $\mathrm{mg}$, where $\mathrm{g}$ is the acceleration due to gravity. There is also a frictional force resisting the motion, which is proportional to the speed of the bob with a coefficient of friction $k$. Using Newton's second law of motion, the equation of motion in the tangential direction can be written as

$$
\mathrm{ml} \ddot{\theta}=-\mathrm{mg} \sin (\theta)-\mathrm{kl} \dot{\theta}
$$

To obtain a state model for the pendulum, consider the state variables as $\mathrm{x}_{1}=\theta$ and $\mathrm{x}_{2}=\dot{\theta}$. Then, the state equations this pendulum system are obtained and given in equation (2). 


$$
\begin{aligned}
& \dot{\mathrm{x}}_{1}=\mathrm{x}_{2} \\
& \dot{\mathrm{x}}_{2}=-\frac{\mathrm{g}}{\mathrm{l}} \sin \left(\mathrm{x}_{1}\right)-\frac{\mathrm{k}}{\mathrm{m}} \mathrm{x}_{2}
\end{aligned}
$$

Sometimes it is instructive to consider the pendulum equation where the frictional resistance is neglected by setting $\mathrm{k}=0$.

Considering the torque as a control input in the resultant equation can be modified and given in equation (3) by substituting $\mathrm{k}=0$.

$$
\begin{aligned}
& \dot{\mathrm{x}}_{1}=\mathrm{x}_{2} \\
& \dot{\mathrm{x}}_{2}=-\frac{\mathrm{g}}{1} \sin \left(\mathrm{x}_{1}\right)+\frac{1}{\mathrm{ml}^{2}} \mathrm{~T}
\end{aligned}
$$

This system can be modeled in terms of nonlinear functions $\mathrm{f}$ and $\mathrm{b}$ i.e., $\mathrm{f}$ is a nonlinear function involving $\mathrm{x}_{1}$ and $\mathrm{x}_{2}$ variables $\left(\sin \left(\mathrm{x}_{1}\right)\right)$.

$$
\dot{\mathrm{x}}(\mathrm{t})=\mathrm{f}(\mathrm{x})+\mathrm{b}(\mathrm{x}) \mathrm{u}
$$

\section{Sliding Mode Control}

The Sliding Mode Control (SMC) design consists of two basic steps (i) defining a sliding surface along which the system can slide to its desired final states .The system trajectories are constrained onto a surface in the state space called the sliding surface. (ii) Designing a control law. The function of the control law is to attract the system trajectories towards sliding surface in finite time and also to keep the trajectories on the sliding surface. The control law consists of linear and nonlinear parts.

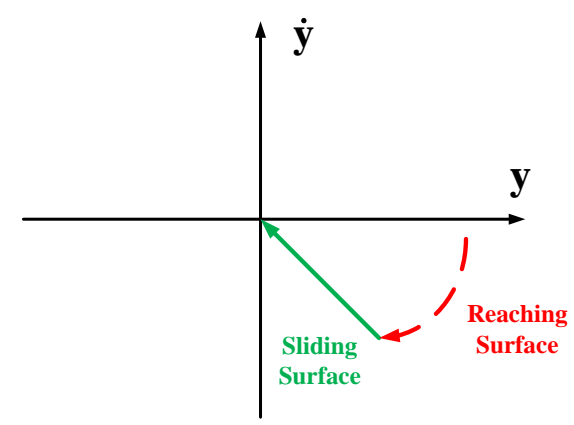

Fig. 2. Sliding Mode Control

Based on the sliding surface design the SMC strategies can be classified as first, second and higher order sliding mode approaches. The higherorder sliding mode control (HOSMC) [3],[10], defined as constraining the state trajectory to reach in finite time onto the sliding surface $\mathrm{s}^{\mathrm{r}}$ represented using equation (5).

$$
\mathrm{s}^{\mathrm{r}}=\left\{\mathrm{x} \in \mathrm{R}^{\mathrm{n}}: \mathrm{s}=\dot{\mathrm{s}}=\ddot{\mathrm{s}}=\ldots=\mathrm{s}^{(\mathrm{r}-1)}=0\right\}
$$

here ' $r$ ' is order of SMC. The advantages of higher order sliding mode control strategy [10]-[12] includes (i) robustness to matched uncertainty (ii) Chattering reduction (iii) Higher convergence accuracy etc,. In this study, onlyFOR second-order sliding mode control (SOSMC) and specially twisting and super-twisting algorithms are demonstrated in simulation in MATLAB environment for the control of the simple pendulum.

\section{Relative Degree}

Relative degree to $r$ means, in a simplified way, that the control input " $u$ " first appears explicitly only in the $r^{\text {th }}$ full time derivative of " $s$ ". Relative degree depends on the choice of system output, here we consider sliding surface $\mathrm{S}$ as the system output. It is used to classify the sliding mode control strategies and also signifies the effectiveness of that control strategy.

\section{1) First Order Sliding Mode Control (FOSMC):}

Consider the nonlinear model of the simple pendulum system as described in the equation (4). Design of FOSMC involves two important steps: (a) defining properly the sliding surface, (b) Design of control law so that system trajectories attracted towards the sliding surface.

(a)Defining Sliding Surface:

The sliding surface can be defined using the equation (6).

$$
\mathrm{S}=\left\{\mathrm{x} \in \mathrm{R}^{\mathrm{n}}: \mathrm{s}=0\right\}
$$

Where $\mathrm{x} \in \mathrm{R}^{\mathrm{n}}$ is the state vector and on sliding surface trajectories obey " $\mathrm{s}=0$ ".

\section{(b) Designing Control Law:}

The control law of the FOSMC is expressed as follows:

$$
\mathrm{u}=\mathrm{u}_{\mathrm{eq}}+\mathrm{u}_{\mathrm{d}}
$$

with $u_{e q}$ is the equivalent control and $u_{d}$ is the discontinuous control. The discontinuous control signal is selected so as to bring the state trajectories towards the switching surface or sliding surface [1][3]. The equivalent control is a control action necessary to maintain an ideal sliding motion in other words it ensures the state trajectory to remain on the switching surface $\mathrm{s}=0$ during the sliding mode.

The equivalent control $u_{e q}$ is obtained by setting $\left.\dot{\mathrm{s}}(\mathrm{x}, \mathrm{u}, \mathrm{t})\right|_{\mathrm{u}=\mathrm{u}_{\mathrm{eq}}}=0 \quad$ where $\dot{\mathrm{s}}=\mathrm{f}(\mathrm{x}, \mathrm{u}, \mathrm{t}) . \quad$ The discontinuous control can be defined as

$$
\mathrm{u}_{\mathrm{d}}=-\mathrm{ksign}(\mathrm{s})
$$

2) Higher Order Sliding Mode Control (HOSMC): Higher order sliding mode control not only removes some of the fundamental limitations of the traditional approach but also provides improves 
tracking accuracy under sliding motion. The $\mathrm{r}^{\text {th }}$ order sliding mode is defined in the equation (5).

2.1) Second Order Sliding Mode Control:

The second order sliding mode controller forcing the system state trajectories in finite time onto the second order sliding surface that defined using equation (5) on substitution of $r=2$.

i) Defining Sliding Surface:

In second order sliding mode all the trajectories will converge in finite time to the origin $\mathrm{s}=\dot{\mathrm{s}}=0$ of the phase plane $(\mathrm{s}, \dot{\mathrm{s}})$ [7]-[10]. Here, the sliding surface can be defined using the equation (9).

$$
\mathrm{s}=\left\{\mathrm{x} \in \mathrm{R}^{\mathrm{n}}: \mathrm{s}=\dot{\mathrm{s}}=0\right\}
$$

In matrix representation form, sliding surface " $\mathrm{s}$ " is defined as $s(x)=S x$, here $x$ is a state vector and $\mathrm{S} \in \mathrm{R}^{\mathrm{n}}$ is switching function matrix. The switching function, 's', its first derivative are continuous and second derivative is discontinuous .

$$
\dot{\mathrm{s}}=\frac{\partial \mathrm{s}}{\partial \mathrm{x}} \dot{\mathrm{x}}=\frac{\partial \mathrm{s}}{\partial \mathrm{x}}(\mathrm{f}(\mathrm{x})+\mathrm{b}(\mathrm{x}) \mathrm{u})
$$

Differentiating the sliding variable $\mathrm{s}$ twice yields to the following relation:

$$
\begin{aligned}
& \ddot{\mathrm{s}}=\frac{\partial \dot{\mathrm{s}}}{\partial \mathrm{x}}(\mathrm{f}(\mathrm{x})+\mathrm{b}(\mathrm{x}) \mathrm{u})+\frac{\partial \dot{\mathrm{s}}}{\partial \mathrm{u}} \dot{\mathrm{u}} \\
& \ddot{\mathrm{s}}=\alpha(\mathrm{x})+\beta(\mathrm{x}) \dot{\mathrm{u}}
\end{aligned}
$$

It is assumed that if $|\mathrm{s}(\mathrm{x})|<\mathrm{s}_{0}$,

$$
|\alpha(x)|<\phi, 0<\Gamma_{\mathrm{m}} \leq \beta(\mathrm{x}) \leq \Gamma_{\mathrm{M}}
$$

Where $\phi, \Gamma_{\mathrm{m}}$ and $\Gamma_{\mathrm{M}}$ are positive constants.

ii. a) Control Law::Twisting Algorithm:

The twisting (TW) algorithm, one of the first known second order sliding mode algorithms, ensures a finite time convergence of the state trajectory to the origin of the phase plane $(s, \dot{\mathrm{s}})$ after executing a certain number of rotations around origin shown in Fig. 3., and the circulation is due to the switch in control law. This algorithm mainly consists of only discontinuous control and described using equation (13). The sufficient conditions that ensure the finite time convergence of trajectories to the second order sliding surface are given in equation (14).

$$
\begin{gathered}
\dot{\mathrm{u}}_{\mathrm{TW}}=\left\{\begin{array}{c}
-\mathrm{u} \text { if }|\mathrm{u}|>\mathrm{u}_{\max } \\
-\mathrm{k}_{\mathrm{m}} \operatorname{sign}(\mathrm{s}) \text { if } \mathrm{s} \dot{\mathrm{s}} \leq 0 \text { and }|\mathrm{u}| \leq \mathrm{u}_{\max } \\
-\mathrm{k}_{\mathrm{M}} \operatorname{sign}(\mathrm{s}) \text { if } \mathrm{s} \dot{\mathrm{s}}>0 \text { and }|\mathrm{u}| \leq \mathrm{u}_{\max }
\end{array}\right. \\
\mathrm{k}_{\mathrm{M}}>\mathrm{k}_{\mathrm{m}}>0, \mathrm{k}_{\mathrm{m}}>\frac{4 \Gamma_{\mathrm{M}}}{\mathrm{s}_{0}}, \mathrm{k}_{\mathrm{m}}>\frac{\phi}{\Gamma_{\mathrm{m}}}, \Gamma_{\mathrm{m}} \mathrm{k}_{\mathrm{M}}-\phi>\Gamma_{\mathrm{M}} \mathrm{k}_{\mathrm{m}}+\phi
\end{gathered}
$$

ii. b) Control Law :: Super-Twisting Algorithm:
Super-Twisting (STW) algorithm is also a second order SMC strategy that stabilizes systems in finite time and also improves the tracking accuracy. The associated switching surface is shown in Fig. 4. In Super-Twisting algorithm the control law " $\mathrm{u}_{\mathrm{ST}}$ " is continuous with respect to time.

The control law and the corresponding sufficient conditions are given in equations (15) and (16).

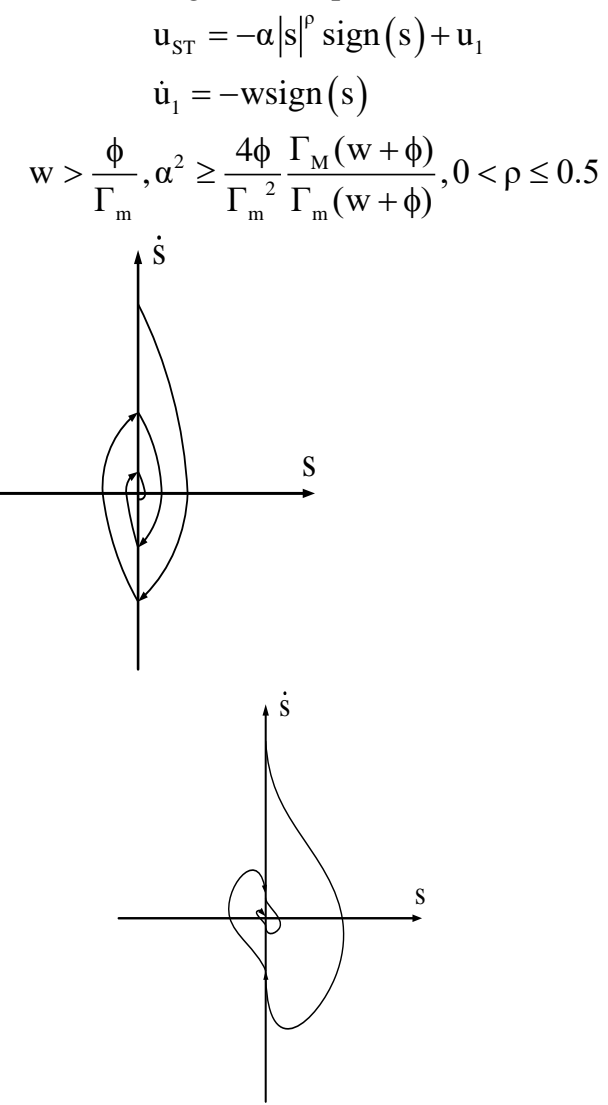

Fig. 3.

Fig. 4.

\section{Results and Discussion}

The Simple Pendulum mathematical model is described using equation (17).

$$
\ddot{\theta}=-0.25 \sin (\theta)+\mathrm{u}
$$

Where $\theta$ is Angular displacement, $\dot{\theta}$ is Angular velocity and $\mathrm{u}$ is Input torque.

a) Using First Order Sliding Mode Control: The computation of first order sliding mode controller for this simple pendulum is enumerated as follows.

1) Defining Sliding Surface for Simple Pendulum: Let us consider Sliding surface be

$$
\mathrm{s}=\dot{\theta}+\theta
$$

And the first time derivative of the Sliding surface is given by equation (19)

$$
\dot{\mathrm{s}}=-0.25 \sin (\theta)+\dot{\theta}+\mathrm{u}
$$


2) Designing Control Law to Simple Pendulum:

In First Order Sliding Mode Control, the control action consists of two parts that is equivalent control $\mathrm{u}_{\mathrm{eq}}$ and discontinuous control $\mathrm{u}_{\mathrm{d}}$. The linear part (equivalent control) of the control strategy is obtained by equating $\dot{\mathrm{s}}=0$. Discontinuous control is considered as $\mathrm{u}_{\mathrm{d}}=-\mathrm{ksign}(\mathrm{s})$, where $\mathrm{k}=0.75$.

The effectiveness this control strategy is verified in simulation for this pendulum system under different initial conditions [0.5 0.2$],[0.6$ $0.3],\left[\begin{array}{ll}0.7 & 0.4\end{array}\right]$ and [ 0.80 .5$]$. The history of angular displacement, control signals with time are plotted under these perturbed conditions and shown in Fig. 5. and Fig. 6. The angular displacement and angular velocity state-space trajectory is shown in Fig. 7. It is observed from these figures that when this pendulum system is subjected to the perturbation, these variables are returning to the original state.

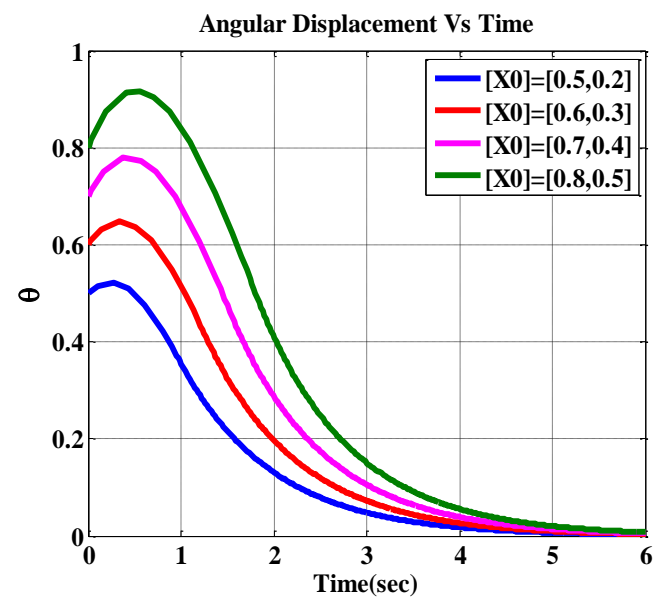

Fig. 5. Angular Displacement versus Time Using FOSMC

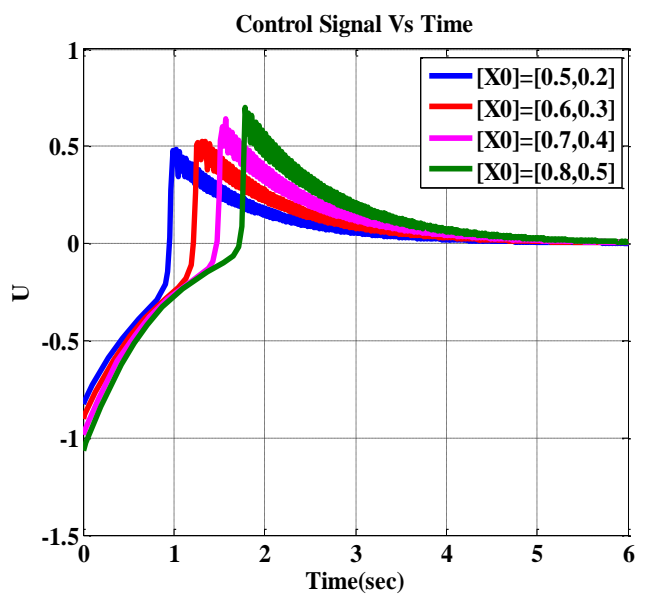

Fig. 6. Control Signal versus Time Using FOSMC

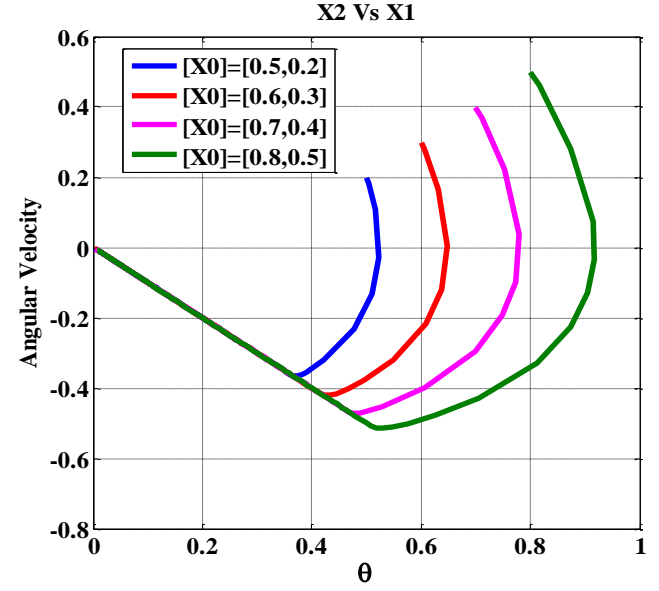

Fig. 7. Angular Velocity versus Angular Displacement Using FOSMC

b) Using Second Order Sliding Mode Control:

The sliding surface is defined as

$$
\mathrm{s}=\dot{\theta}+\theta
$$

And the first time derivative of the Sliding surface is given by equation (21)

$$
\dot{\mathrm{s}}=-0.25 \sin (\theta)+\dot{\theta}+\mathrm{u}
$$

And the second time derivative of the sliding surface is given in equation (22)

$$
\ddot{\mathrm{s}}=-0.25 \dot{\theta}(\cos (\theta))-0.25 \mathrm{~s}(\sin (\theta))+\mathrm{u}+\dot{\mathrm{u}}
$$

These equations are used to derive the second order SM control law. The equation (22) is compared with equation (11), to obtain the boundaries of $\ddot{\mathrm{s}}$ ( $\phi, \Gamma_{\mathrm{m}}$ and $\Gamma_{\mathrm{M}}$ ), that drive the trajectories to the required switching surface. By using the information of sliding surface and $2^{\text {nd }}$ order sliding mode control law, simulations are performed with the two different algorithms (i)Twisting SMC(TSMC), (ii)Super-Twisting SMC(ST-SMC)

\section{i) Twisting Algorithm:}

In twisting algorithm, the controller constants $\mathrm{k}_{\mathrm{m}}, \mathrm{k}_{\mathrm{M}}$ are chosen such that, they ensure the system trajectories convergence in a finite time to the sliding surface. From the sufficient conditions, the constants obtained are $\mathrm{k}_{\mathrm{m}}=0.7, \mathrm{k}_{\mathrm{M}}=7$.

The effectiveness this algorithm is verified in simulation under different initial conditions [0.5

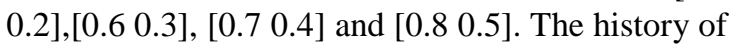
angular displacement, control signals with time are plotted under these perturbed conditions and shown in Fig. 8. and Fig. 9. The trajectory of switching surface and its derivatives is shown in Fig. 10. It is observed from these figures that when this pendulum system is subjected to the perturbation, these variables are returning to the original state. 


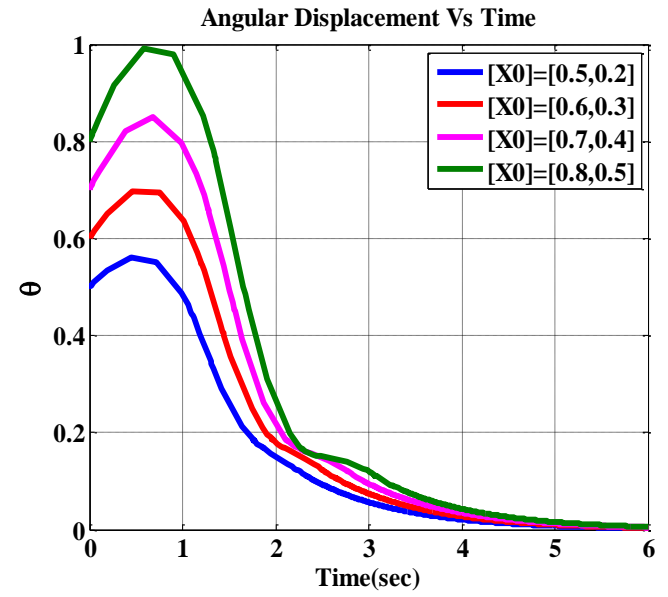

Fig. 8. Angular Displacement versus Time Using TW-SMC

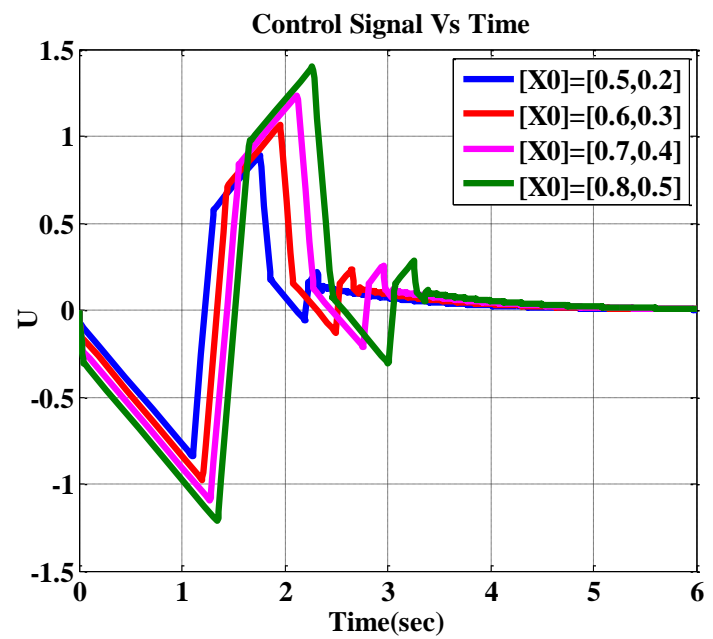

Fig. 9. Control Signal versus Time Using TW-SMC

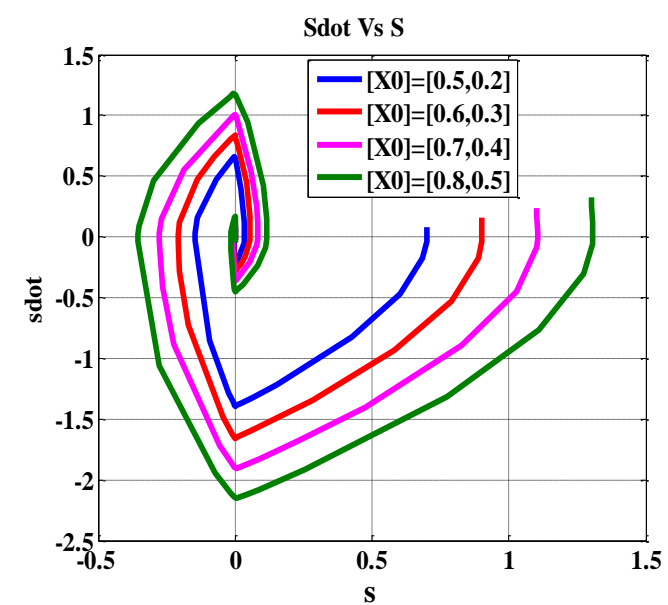

Fig. 10. First derivative of Sliding Surface versus Sliding Surface Using TW-SMC

ii) Super-Twisting Algorithm (STW):

In super twisting algorithm, the controller constant $\alpha, w, \rho$ are chosen such that, they will ensure the finite time convergence of state trajectories to the switching surface. From the sufficient conditions, the constants obtained are $\alpha=1.5, \mathrm{w}=3, \rho=0.5$.

The effectiveness of super twisting algorithm is verified in simulation under different initial conditions $\quad\left[\begin{array}{ll}0.5 & 0.2\end{array}\right],\left[\begin{array}{ll}0.6 & 0.3\end{array}\right],\left[\begin{array}{ll}0.7 & 0.4\end{array}\right]$ and [0.8 $0.5]$. The history of angular displacement, control signals with time are plotted under these perturbed conditions and shown in Fig.11. and Fig. 12. The trajectory of switching surface and its derivatives is shown in Fig. 13. It is observed from these figures that when this pendulum system is subjected to the perturbation, these variables are returning to the original state.

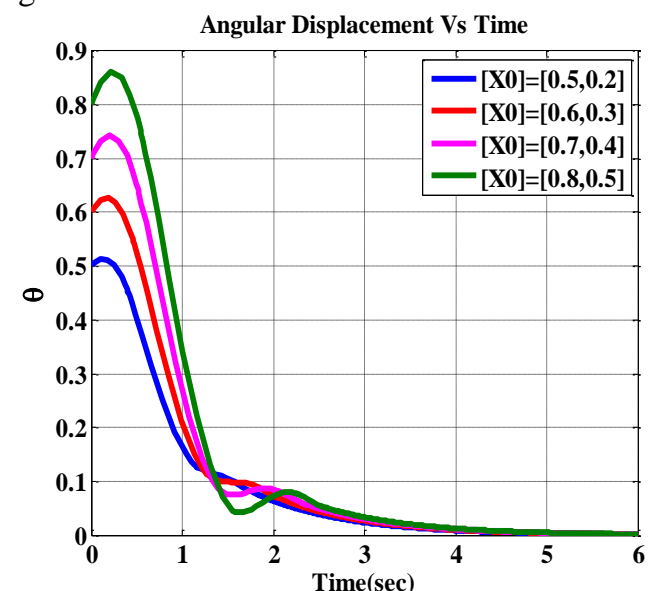

Fig. 11. Angular Displacement Versus Time Using

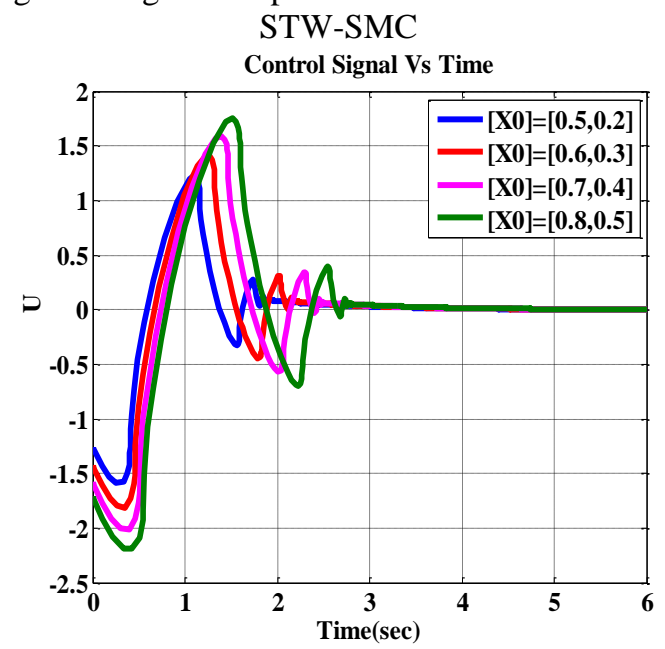

Fig. 12. Control Signal versus Time Using STWSMC 


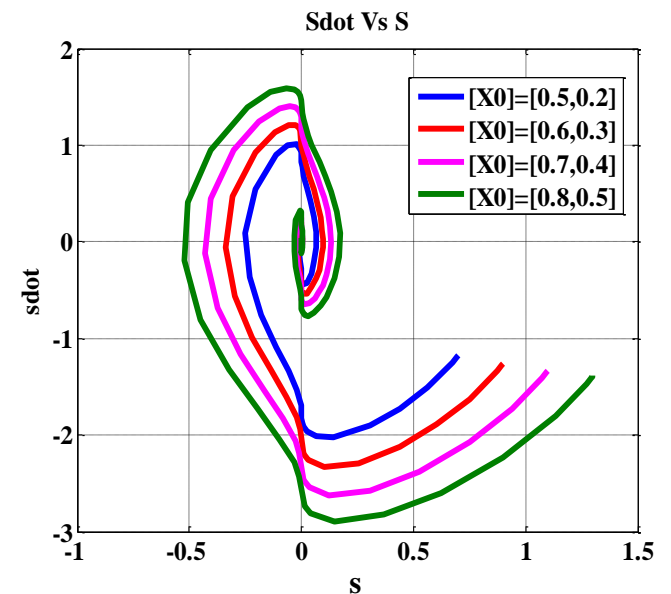

Fig. 13. First derivative of Sliding Surface versus Sliding Surface Using STW-SMC

\section{Conclusions}

This paper presents first order sliding mode controller for a simple pendulum. The second order sliding mode controllers are also designed using twisting and super-twisting algorithm. Using these controllers simulation studies are performed under different initial conditions. Angular displacement, control signals and trajectories are plotted. It is observed from the simulation results that, steady state convergence time for angular displacement is less in twisting SMC strategy and further less in Super-Twisting SMC strategy than compared to First order sliding mode control strategy.

\section{References}

1. Hassan K.Khalil, "Non-Linear Systems", Pearson, (2002)

2. Chrisrtopher Edwards and Sarah K. Spurgeon, "Sliding Mode Control: Theory and Applications", Taylor \& Francis, (1998)

3. Vadim Utkin, Jurgen Guldner, Jingxin Shi, "Sliding Mode Control in ElectroMechanical Systems", Taylor \& Francis Group, (2009)

4. S.V.Emel'yanov, "Design of variable structure control systems with discontinuous switching function", EngCybern,1,pp. 156-160,(1964).

5. U. Itkis, "Control Systems of Variable Structure", Wiley, New York, (1976).

6. J. Slotine, \& S.Sastry, "Tracking control of non-linear systems using sliding surfaces, with application to robot manipulators", International Journal of Control, 83, 465492(1983).

7. S. V. Emel'yanov, "Automatic control systems of variable structure", Nauka, Moskow, (1967).

8. J.-J. E. Slotine, "Sliding controller design for nonlinear systems", Int. J. of Control, Vol. 40, No.2,(1984).
[9] G. Bartolini, T. Zolezzi, "Control of nonlinear variable structure systems", J. Math. Anal. Appl, 118, pp. 42-62, (1986).

[10] Levant, "Sliding order and sliding accuracy in sliding mode control.", Int. J. Control, 58(6):1247.1263, (1993)

[11] S.V. Emel'yanov, S.K. Korovin, and A. Levant. "High order sliding modes in control systems. Computational mathematics and modeling", 7(3):294.318, (1996).

[12] V.I. Utkin "Sliding modes in control and optimization", Springer-Verlag, (1992). 